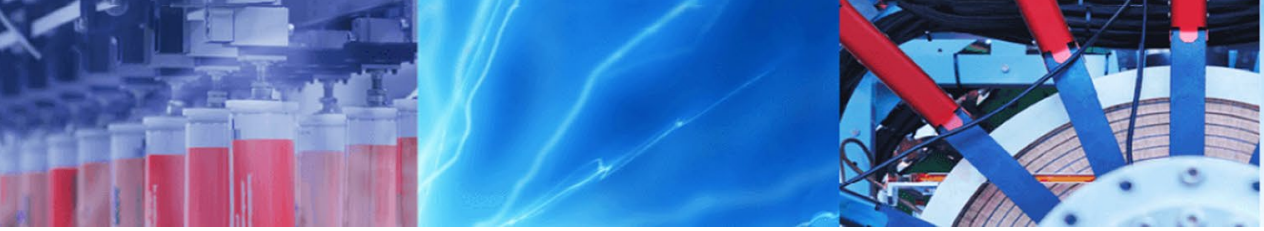

Research Article

\title{
Optimization and characterization of novel injection molding process for metal matrix syntactic foams
}

\author{
Myranda Spratt $^{1}$ (D) . Joseph W. Newkirk ${ }^{1}$ (D)
}

Received: 8 July 2020 / Accepted: 29 October 2020 / Published online: 21 November 2020

(c) Springer Nature Switzerland AG 2020

\begin{abstract}
Metal matrix syntactic foams are particulate composites comprised of hollow or porous particles embedded in a metal matrix. These composites are difficult to manufacture due primarily to the lightweight, relatively fragile filler material. In this work, an injection molding process was developed for metal matrix syntactic foams. First, an aqueous binder was optimized for low-pressure injection molding. A mixture model was used to optimize the composition of the binder to achieve the highest relative density. The model predicted the maximum relative density was at a binder composition (in vol.\%) of $7 \%$ agar, $4 \%$ glycerin, and $89 \%$ water. Second, this binder was used to manufacture copper matrix syntactic foams with $0,5,10$, and 15 vol.\% porous silica as the filler material. The solids loading for these compositions decreased with increasing filler material from 55 to 44 vol.\%, likely due to binder filling the pores in the porous silica particles. Finally, the sample quality after injection molding was characterized. Only $0.11 \pm 0.06$ vol.\% carbon remained in the samples. Silica particles were well-dispersed in the samples after sintering, and they did not appear to be fractured. The specific strength of the copper matrix material increased with increasing porous silica additions.
\end{abstract}

Keywords Metal-matrix composites $\cdot$ Metal foams $\cdot$ Injection molding $\cdot$ Molding compounds

\section{Introduction}

Lightweight structural composites are in high demand in certain industries, especially in aerospace. These materials are generally two-phase composites of some kind. For example, an aluminum-silicon carbide composite increases the strength of the aluminum and slightly increases the density for an overall increase in the specific strength and stiffness. Other types of composites combine a matrix and air-foams sacrifice strength for drastically decreasing the density of the material. Syntactic foams combine the best of both types of composites. Metal matrix syntactic foams (MMSF) are 3-phase particulate composites where hollow particles are suspended in a metal matrix [1]. The pores in MMSFs are encased in the hollow spheres, which allows for both tight control over the density and pore size of the material and restricts the porosity from interacting with the matrix material directly. Rohatgi et al. [2] summarized the typical compression properties of MMSF in their review paper. MMSF exhibit other properties as well as a result of being both foams and composites. For example, Mondal et al. [3] found that the wear rate of an aluminum-cenosphere MMSF was comparable to an Al-SiC composite at low loads. Dou et al. [4] found that MMSFs might be useful electromagnetic shielding materials.

There are several methods of manufacturing MMSFs and several challenges in doing so. Stir casting and pressure infiltration are the most popular methods [2]. Powder metallurgy routes such as injection molding and press and sinter are less common. The main challenge in manufacturing these materials is in incorporating the filler into the matrix without segregation, sphere fracture, or negative

Myranda Spratt, MSpratt.School@gmail.com; Joseph W. Newkirk, jnewkirk@umsystem.edu | ${ }^{1}$ Materials Science Department, Missouri University of Science and Technology, Rolla, MO, USA.

SN Applied Sciences (2020) 2:2048 | https://doi.org/10.1007/s42452-020-03791-y 
chemical interactions between the matrix and filler. In their review of the types of hollow spheres used in the literature, Szlancsik et al. [5] found that the hollow particles, or filler, are usually a glass or ceramic material, though metal particles and expanded clay have also been utilized. In particular, fly ash cenospheres remain a popular filler as they would otherwise be a waste-product and can withstand higher temperatures than glass fillers $[2,6,7]$, The low density filler materials float in molten metal which can cause density gradients. The hollow particles can fracture during processing as well [8]. Material compatibility is another challenge. The melting temperature of most glasses, other than pure silica, is less than the processing temperature of many metals. Diffusion of the matrix material into the filler and vice versa can also occur, which can cause chemical reactions to occur $[9,10]$. In glasses, this can also cause the melting temperature of the filler to decrease. Oxide ceramics might not wet strongly with the matrix. The two powders should bond strongly so that load partitioning can occur [11]. Neville and Rabiei [12] worked around these issues by using metal hollow particles as the filler. Májlinger et al. [13] studied the effect of using multiple types of particles in the same material. Lehmhus et al. [14] compared hollow glass to cenosphere filler in a steel alloy (316L) matrix.

A key part of MMSF research is the development of novel techniques to improve the manufacturing of MMSF materials. For example, Weise et al. [15] and Yang et al. [16] used similar methods to ensure an even distribution of hollow particles during infiltration of the metal matrix. They sintered the filler before infiltration-Weise et al. [15] using glass filler and Yang et al. [16] using ceramic spheres. Augmentation of a manufacturing technique is one method of working around the manufacturing weaknesses of MMSF materials. Other researchers, such as Orbulov [17] with pressure infiltration, optimize a manufacturing process for MMSF materials. Another method is to use a novel manufacturing technique such as done by Shiskin et al. [18]. They sputter-coated the hollow spheres with copper then used spark plasma sintering to densify the parts. Further, a manufacturing technique that mitigates many of the manufacturing issues can be selected.

Injection molding is one of those techniques that need little process optimization to effectively make MMSF materials. Hollow particle flotation is not an issue in injection molding as the powder is held in place by a binder. Particle fracture can still be an issue, but gentle comminution and injecting procedures can be used. Weise et al. $[19,20]$ used injection molding to produce an iron alloy matrix with hollow glass microsphere syntactic foams. They were able to sinter the iron matrix to the point where little matrix porosity remained. However, the glass did appear to soften and wick into residual porosity during sintering. This is a material compatibility problem solved by using ceramic powders rather than glass ones. Hollow ceramic powders that are small enough to use in injection molding and of good quality are difficult to find without requesting specialty powders. Injection molding requires fine powders to form stable slurries that can be injected into molds. As described by Szlancsik et al. [5], most hollow ceramic powders are in the $1-10 \mathrm{~mm}$ size range, not the $<45 \mu \mathrm{m}$ range that is typically used for injection molding processes.

Injection molding requires careful selection of powders and a compatible binding agent. The powder shape and size will influence both sintering and slurry rheology. Fine powder sinter better than coarse powders due to their increased surface area. This increase in surface area may negatively affect slurry fluidity. Bimodally distributed powders achieve higher green densities as small particles fit between larges ones and fill space. It is mathematically appropriate to have the fine particles in the bimodal distribution be $1 / 7$ th of the coarse particles. For MMSFs, this might mean that the matrix particles should be, on average, 7 times smaller than the hollow particles, or vice versa. The choice of binder will determine the binder removal and burnout procedure. The binder usually leaves some carbon or oxides behind, so minimizing the amount of binder needed to create a flowable slurry is necessary.

Aqueous binders leave very little material leftover after the water backbone has been removed. One such binder is a water-based gel is comprised of agar, glycerin, and water [21]. The basic formula for this binder is agar and water which, when heated, forms a polysaccharide gel. The glycerin in the formula acts as a gel strengthener. The binder has similar rheological properties to a thermoplastic according to Labropoulos et al. [22]. It has an added benefit of being more environmentally friendly than waxy binders as usually more than $90 \%$ of the binder is water. The general formula for this binder was the subject of or used in several patents [23-25]. It was also produced commercially (Honeywell Powderflo Technologies, for example). Chen et al. [26] produced NiTi foams with an agar binder, though they used sucrose as the gel strengthener rather than glycerin.

The goals of this work were to develop an aqueous binder for low-pressure injection molding of metal matrix syntactic foams and successfully manufacture a copper matrix syntactic foam using that binder and process. The composition of the agar-glycerin-water binder was optimized to obtain the highest green and sintered relative density in a bronze syntactic foam. The relative density was selected as a simple way to check the quality of the parts. This optimized binder was then used to injection mold copper matrix syntactic foams with a porous silica filler. Four samples were made with an increasing volume fraction of filler. The quality of the sintered samples was 
analyzed. The MMSFs were tested in flexure to determine the mechanical properties and compared to the literature.

\section{Material and methods}

\subsection{Powder characterization}

Powder characterization was performed on the syntactic foam materials to verify morphology, and particle size distribution. Table 1 lists the syntactic foam materials used in this experiment with the manufacturer-supplied chemistry and density. The particle size distribution was characterized via particle measuring and counting from SEM images. Each powder was dusted over the surface of a carbon dot on a flat specimen holder. The powder was then mounted into an ASPEX scanning electron microscope (SEM). The ASPEX has an automated feature analysis (AFA) that will identify and measure inclusions or particles. At least 10,000 particles were measured for each powder, except the two glasses. The borosilicate glass and porous silica powders could not be distinguished from the noise in the ASPEX and so the particle 'features' were instead analyzed by manual measurement. This was done using FIJ (Fiji Is Just ImageJ) software. The AFA included the aspect ratio of each particle, which (along with visual inspection of the SEM images) was used to determine morphology.

\subsection{Binder development}

In this study, an aqueous binder was developed using a mixture model. The model is discussed in the calculation section of this paper. The binder components were deionized water, agar, and glycerin. The mixture model requires 10 samples with different binder compositions to optimize the composition. The solids loading remained constant at 55 vol.\%, and the solid composition was held constant as well. The target sintered sample was a $30 / 70$ volume ratio of hollow borosilicate spheres to bronze matrix. Specimens were made in $50 \mathrm{~g}$ batches by manually mixing the binder and the solid powders. To make the binder, boiling water was mixed with the glycerin and agar in the appropriate combinations. The mixture was stirred until all the dry ingredients dissolved into the solution, about $5 \mathrm{~min}$. The solid components were heated to $100^{\circ} \mathrm{C}$ then mixed into the binder. Each specimen was poured into a $50 \times 20 \times 15 \mathrm{~cm}$ silicone mold. Three repetitions were done for each sample, and all specimens were made and measured in a completely random order. Sample post-process characterization is discussed in Sect. 2.4.

A binder-only trial in the injection mold machine was done to determine the rate of water evaporation during use. In this test, the binder components were added to the reservoir. The reservoir was then heated, while mixing, to $80^{\circ} \mathrm{C}$. Samples were injection molded every 30 minutes from 30 to 120 minutes, with 5 specimens per sample. The specimens were dried at $120^{\circ} \mathrm{C}$ for 12 hours. The mass of the specimens before and after drying was measured and used to find the liquid loss of the binder. These values were then compared over time to find the change in mass loss over time.

\subsection{Injection molding}

A Peltsman MIGL-28 was used to injection mold the samples in this experiment. This low-pressure injection mold machine includes a feedstock reservoir that also compounds of the feedstock before injection molding. A single paddle mixes the slurry in the reservoir at $58 \mathrm{rpm}$. The reservoir temperature was set at $80^{\circ} \mathrm{C}$ for this experiment. Gas pressure is applied to the reservoir during molding which pushes material through a heated tube to the mold. The tube and orifice ring were set to $85^{\circ} \mathrm{C}$. The gas pressure was set at $67 \mathrm{kPa}$. The pressure was held for $10 \mathrm{~s}$ per specimen. The specimens were demolded after approximately $30 \mathrm{~s}$. The mold used in this experiment was a watercooled iron alloy rectangular mold. The dimensions were $6.21 \times 0.99 \times 0.66 \mathrm{~cm}$.

Four compositions were injection molded in this study. Table 2 shows the volume fraction of each component that was added to the reservoir, out of the total volume. Thirty
Table 1 Details provided by the manufacturer on each metal and ceramic powder used in this experiment

\begin{tabular}{llll}
\hline Material & Supplier & Chemistry & Density (g/cc) \\
\hline Borosilicate glass & MO-SCl Corp. & $70-85 \% \mathrm{SiO}_{2}$ & 0.15 \\
& & $10-15 \% \mathrm{~B}_{2} \mathrm{O}_{3}$ & \\
& & $5-10 \% \mathrm{Na}_{2} \mathrm{O}$ & \\
& & $2-5 \% \mathrm{Al}_{2} \mathrm{O}_{3}$ & \\
Porous silica (P-S) & MO-SCl Corp. & $\mathrm{SiO}_{2}$ & 1.475 \\
Copper & Royal Metal Powders Inc. & $99.8 \%$ copper & 8.94 \\
& & $0.06 \%$ hydrogen loss & \\
Bronze & Royal Metal Powders Inc. & $88.46 \%$ copper & 8.73 \\
& & $11.3 \%$ tin & \\
& & $0.24 \%$ phosphorous & \\
\hline
\end{tabular}


Table 2 The composition of each slurry in the injection molding reservoir before injection molding is listed, all units in vol.\%

\begin{tabular}{lllll}
\hline Component & 0\% P-S (\%) & 5\% P-S (\%) & 10\% P-S (\%) & $\begin{array}{l}15 \% \text { P-S } \\
(\%)\end{array}$ \\
\hline Agar & 3 & 3 & 4 & $4 \%$ \\
Glycerin & 2 & 2 & 2 & 2 \\
Water & 40 & 44 & 50 & 50 \\
Binder total & 45 & 49 & 56 & 56 \\
P-S & 0 & 3 & 4 & 7 \\
Copper & 55 & 49 & 40 & 38 \\
Solids total & 55 & 51 & 44 & 44 \\
\hline
\end{tabular}

specimens were injection molded for each sample. Each sample was prepared by mixing the binder on a hot plate, then adding the prepared binder to the pre-warmed IM reservoir. The powders were added to the reservoir preheated to $100^{\circ} \mathrm{C}$. For some samples, extra binder was added to decrease slurry viscosity. After the slurry viscosity was adjusted, it was mixed for at least $1 \mathrm{~h}$ before injection molding.

\subsection{Sample post-processing and characterization}

After injection molding, the 'wet' samples were placed in a furnace at $120^{\circ} \mathrm{C}$. Samples were dried for at least $12 \mathrm{~h}$ before sintering. The 'dry' specimens underwent debinding and sintering in an atmospheric furnace with an attached retort. Debinding was done in air at $450^{\circ} \mathrm{C}$ for $1 \mathrm{~h}$. A steel plate was placed in the furnace beside the samples to help getter escaping carbon and oxygen. Sintering was done directly after debinding by first dwelling at $450^{\circ} \mathrm{C}$ for $1 \mathrm{~h}$ in flowing argon to remove any remaining oxygen. Then, the temperature was increased at a rate of $120^{\circ} \mathrm{C} / \mathrm{h}$. Samples remained under flowing argon throughout the sintering time. Bronze matrix samples were held at $900^{\circ} \mathrm{C}$ for $1 \mathrm{~h}$. Copper matrix samples were held at $1000^{\circ} \mathrm{C}$ for $10 \mathrm{~h}$. Samples were removed from the furnace after cooling.

Characterization included density measurements and microstructural evaluation. The geometric density of each specimen was measured after each processing stage (wet, dry, and sintered). The geometric density calculation is mass over volume, where volume was measured by calipers. The mass was measured using a laboratory balance. The sintered density was also measured by Archimedes' method (ASTM C373-18 [27]). The vacuum method was used as described in the standard. Theoretical density at each stage of processing was done by rule of mixtures using the measured amounts of each component added to the injection molding reservoir for each sample. Specimens were prepared for microstructure observation by mounting specimens in bakelite and polishing. The ASPEX
SEM was used for standardless energy-dispersive $\mathrm{x}$-ray spectroscopy (EDS) mapping of each sample. The automated feature analysis function was used on the polished surface of one specimen from each sample as well. This analysis measured the chemistry (using EDS), area, and average diameter of 10,000 features. The features were sorted by chemistry. The total area of the features and the total area observed were used to calculate the total area percent of features in the specimen.

The samples were the correct size and shape for 3-point bend testing. ASTM C1161-18 [28] was used as a guide for this test, with test configuration B. Fifteen specimens were tested for each sample. A fully articulating fixture was used. A strain rate of $0.5 \mathrm{~mm} / \mathrm{min}$ was used. Pre-loading was not done for these tests. The samples were ductile, not brittle, and there was no clear yield or fracture point. Thus, the $0.2 \%$ offset flexural yield stress was calculated from the stress-strain curves. Visual Basic for Excel (VBA) code was used to remove the toe region from each data set and determine the $0.2 \%$ offset yield stress. The VBA code measured the $0.2 \%$ offset yield stress by first finding the slope of the linear elastic region. Then, a line was created offset by $0.2 \%$ from the $x$-axis. The intersection between the new line and the stress-strain curve was taken as the $0.2 \%$ offset yield stress.

\section{Calculation}

The agar-glycerin-water binder used in this experiment was developed using a 3-component simplex mixture model. The wet, dry, and sintered relative density responses to altering the ratios of agar to glycerin to water were modeled. Seven compositions were used to create the model, and three compositions were used to check the model. The overall composition of each sample was 45 vol.\% binder, 33 vol.\% bronze, and 22 vol.\% hollow borosilicate glass. Each sample had three replications. The 30 specimens were made and measured completely randomly. JMP software was used to design the experiment and create the response surface graphs.

The experiment was designed by first selecting the lower bound of each component. The lower limit of agar was determined after tests showed that agar does not gel with sufficient strength below $4 \%$. The glycerin was only a gel strengthener so the lower limit was set at $0 \%$. Finally, after about $15 \%$ agar, the agar became increasingly difficult to completely dissolve into the water so the lower limit on the water was set at $85 \%$. The standard composition array for the mixture model is shown in Table 3. This table shows three pseudo-components which exist at the corners of the ternary response surface graph. The pseudo-components 
Table 3 The general composition of each sample for the model calculation, all units in vol.\%

\begin{tabular}{lllllllllll}
\hline $\begin{array}{l}\text { Pseudo- } \\
\text { components }\end{array}$ & $1(\%)$ & $2(\%)$ & $3(\%)$ & $4(\%)$ & $5(\%)$ & $6(\%)$ & $7(\%)$ & $8(\%)$ & $9(\%)$ & $\begin{array}{l}10 \\
(\%)\end{array}$ \\
\hline A & 100 & 0 & 0 & 50 & 50 & 0 & 33 & 67 & 17 & 17 \\
B & 0 & 100 & 0 & 50 & 0 & 50 & 33 & 17 & 67 & 17 \\
C & 0 & 0 & 100 & 0 & 50 & 50 & 33 & 17 & 17 & 67 \\
\hline
\end{tabular}

$b_{3}=y_{3}$

$b_{12}=4 y_{4}-2\left(y_{1}+y_{2}\right)$

$b_{13}=4 y_{5}-2\left(y_{1}+y_{3}\right)$

$b_{12}=4 y_{6}-2\left(y_{2}+y_{3}\right)$

$b_{123}=27 y_{7}-12 *\left(y_{4}+y_{5}+y_{6}\right)+3\left(y_{1}+y_{2}+y_{3}\right)$

where $y_{1}$ to $y_{7}$ are the average measured response of samples one through seven.

$S_{L F}^{2}=\frac{1}{r}\left(\sum\left(y_{o i}-y_{p r i}\right)^{2}\right)$

where $y_{o i}$ is the observed average response at the $i$ th checkpoint (samples 8-10), and $y_{\text {pri }}$ is the predicted response at the ith checkpoint.

$F=\frac{S_{L F}^{2}}{S_{e r r o r}^{2}}$

where $i$ is the actual component amount (agar, glycerin, or water), $i_{L B}$ is the lower bound of component $i$, and $x_{i}$ is the amount of the pseudo-component.

$\begin{aligned} \text { Cubic Response Model }= & b_{1} x_{1}+b_{2} x_{2}+b_{3} x_{3}+b_{12} x_{1} x_{2} \\ & +b_{13} x_{1} x_{3}+b_{23} x_{2} x_{3}+b_{123} x_{1} x_{2} x_{3}\end{aligned}$

where $x_{1}, x_{2}$, and $x_{3}$ are the amount of pseudo-components $\mathrm{A}, \mathrm{B}$, and $\mathrm{C}$, respectively, and $b_{1}, b_{2}, b_{3}, b_{12}, b_{13}, b_{23}$, and $b_{123}$ are related to the measured response values as indicated in Eqs. (3)-(9).

$b_{1}=y_{1}$

$b_{2}=y_{2}$ where $S_{\text {error }}{ }^{2}$, the error variance was calculated with Eq. (12).

$S_{\text {error }}^{2}=\frac{S_{\text {pooled }}^{2}}{n}$

where $S_{\text {pooled }}{ }^{2}$ was calculated with Eq. (13) and is the pooled variance of all the data, and $n$ is the number of replications per sample.

$S_{\text {pooled }}^{2}=\frac{1}{n-1} \sum S_{i}^{2}$

where $S_{i}^{2}$ is the variance of the ith sample.
Table 4 The composition of each binder for the model calculation, all units in vol.\%

\begin{tabular}{lllllllllll}
\hline Pure components & $1(\%)$ & $2(\%)$ & $3(\%)$ & $4(\%)$ & $5(\%)$ & $6(\%)$ & $7(\%)$ & $8(\%)$ & $9(\%)$ & $\begin{array}{l}10 \\
(\%)\end{array}$ \\
\hline Agar & 15 & 4 & 4 & 10 & 10 & 4 & 8 & 11 & 6 & 6 \\
Glycerin & 0 & 11 & 0 & 6 & 0 & 6 & 4 & 2 & 7 & 2 \\
Water & 85 & 85 & 96 & 85 & 91 & 91 & 89 & 87 & 87 & 92 \\
\hline
\end{tabular}




\section{Results and discussion}

\subsection{Powder characterization}

The particle size distributions by count, volume, cumulative count, and cumulative volume percent for each of the four powders are shown in Fig. 1. Table 5 shows the 10th, 50th, and 90th percentiles by count (D10, D50, D90, respectively) and average for each powder. The sizes of the copper and porous silica powders were similar. The borosilica and bronze powders appear to have similar sizes from the table, however, the borosilica powder has far more particles between 10 and $20 \mu \mathrm{m}$ than the bronze powder as shown in Fig. 1. The bronze powder was sieved by the manufacture to remove fines, however,
Fig. 1 The particle size distribution by count and volume is shown for the four powders used in this study. (a) Bronze, (b) borosilica, (c) copper, (d) porous silica
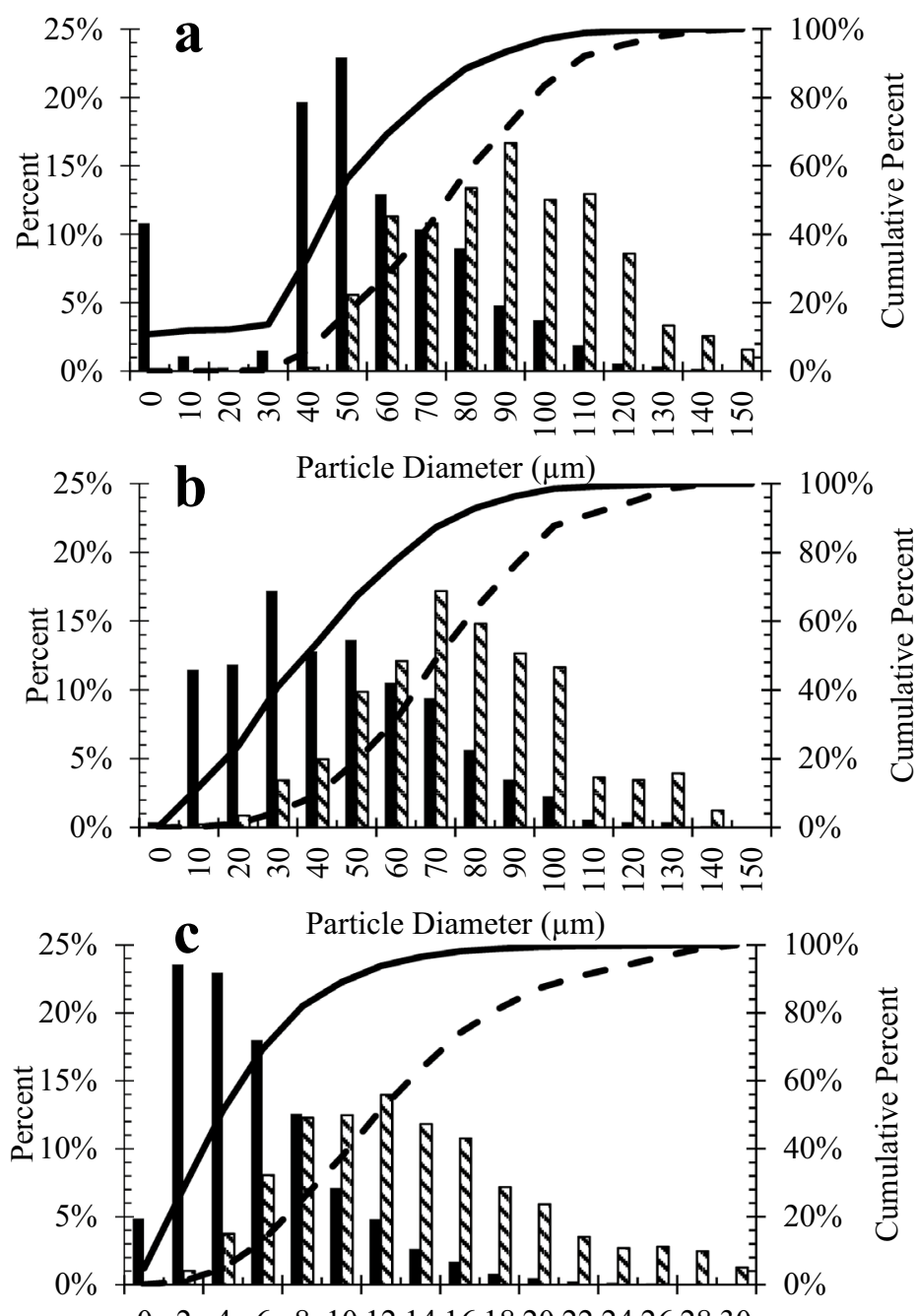

$0 \begin{array}{llllllll}0 & 2 & 4 & 6 & 8 & 1012141618202224262830\end{array}$

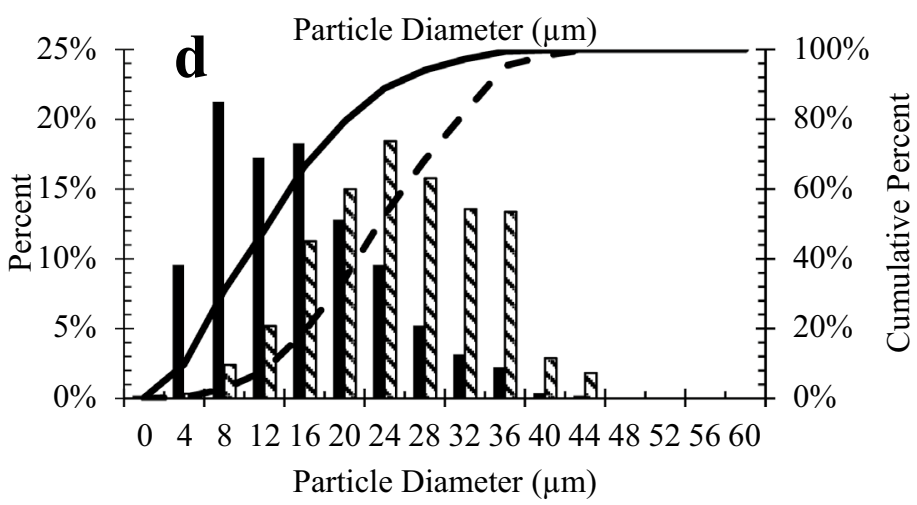

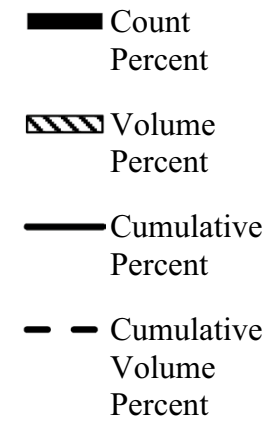


Table 5 The 10th, 50th, 90th percentiles and average particle size in microns for each powder, all units in $\mu \mathrm{m}$

\begin{tabular}{lrrrc}
\hline Powder & D10 & D50 & D90 & Average \\
\hline Bronze & 5 & 56 & 93 & 57 \\
Borosilicate & 20 & 46 & 84 & 50 \\
Copper & 3 & 6 & 13 & 7 \\
P-S & 8 & 16 & 29 & 17 \\
\hline
\end{tabular}

some less than $10-\mu \mathrm{m}$ particles remained ( 10\%) which has skewed the results. Due to the large density difference between the heavy metal powders and the lightweight glass powders, these two composite powders will not stay mixed, if they can be mixed at all. This can be solved by using a binder to facilitate mixing. However, segregation while the binder is liquid will be a concern. In the future, it would be appropriate to sieve out specific particle sizes such that the composite powders are approximately $1: 7$ in size ratio. This will aid in mixing and may help prevent some amount of segregation.

The particle size and morphology of the metal powders will affect how easy they are to process and sinter. Irregular particles sinter marginally better than spherical particles due to their higher surface area, but they are much less flowable. Figure 2 shows example images of each powder that were used to determine the particle size distribution. All the powders used here were spherical, as Fig. 2 shows clearly. The size of the copper powder is suitable for an injection molding and sinter operation. However, the bronze powder is larger than would be typical for a sintering operation, especially a pressure-less sintering operation as was used here. This is one reason that the bronze powder used in the binder optimization procedure was not used for the injection molding process.

\subsection{Binder optimization}

The binder development results include the cubic response equation and graph for each measured response. Table 6 shows the b-values for each response which are applied to Eq. (2) (the cubic response equation) to calculate the response surfaces. Figure 3 shows response surfaces calculated from the cubic response equations for the wet (A), dry (B), and sintered relative density (C), as well as the open porosity after sintering (D). The area of highest relative density appears in the same general area of each graph. The optimized composition was selected to be $7 \%$ agar, $4 \%$ glycerin, and $89 \%$ water. The predicted values for each response given this composition are shown in the last column of Table 6 . The error, pooled variance, lack-of-fit, and F-statistic for the mixture model are shown in Table 7. With a $95 \%$ confidence interval, the tabulated F-statistic is 3.71. All the F-statistics were below this value, so they are considered reasonable estimations of the response. Because the solids loading and solid composition were kept constant across all samples, this experiment should only reveal the trends of the binder. However, the actual density depends strongly on the powder chemistry and particle size. This is especially true of the sintered density. The $b$-values used here are valid only for this experiment, but the trends should be relatively universal.

The binder development work was done using the bronze and hollow borosilicate powders. During sintering, the borosilica glass reacted with the tin in the bronze alloy. Tin oxide and silica inclusions were spotted in the bronze. This material compatibility issue did not invalidate the binder development, as the trends found in this study are considered to be material agnostic. This did prompt the matrix change to copper for the injection molding portion of the study. Further, the melting temperature of the borosilica powder was much lower than the sintering temperature of copper, so the filler material was changed to the porous silica powder.

The binder used in this experiment was described by German and Bose [21] as an aqueous gel useful for injection molding at low temperatures. The binder solidifies below about $50{ }^{\circ} \mathrm{C}$ and should be kept below $100{ }^{\circ} \mathrm{C}$ (boiling) to prevent bubbles from forming in the gel. This binder was chosen for this process specifically for several reasons. First, the Peltsman MIGL-28 IM machine used in this study has a maximum operating temperature of $120^{\circ} \mathrm{C}$, so a relatively low-temperature binder had to be selected. Further, this binder is easily cleaned with friction and warm water and is non-toxic. Finally, this binder is approximately $90 \%$ water, which is both environmentally friendly and easy to remove from the samples. Once the water is removed, the small amount of remaining binder is removed by a binder burnout step. Though pre-made feedstock was not necessary for this experiment, as the IM machine included a mixing apparatus as part of the machine, industrial MIM machines may require pre-mixed feedstock. This binder is also capable of being re-liquidized after comminution with the powders if the water has not been removed. Scale-up of this binder to industrial processes is therefore possible.

Figure 4 shows the amount of water over time in binder-only injection molded samples. During the first 10 to 20 min of the trial, the specimens varied in composition (not shown). The agar and glycerin were added to the water cold, so the agar agglomerated in the reservoir. It took approximately 30 min after reaching the working temperature of $80^{\circ} \mathrm{C}$ for the agar to fully incorporate into the solution. In future trials, the binder was mixed on a hotplate before adding it to the reservoir to prevent this 

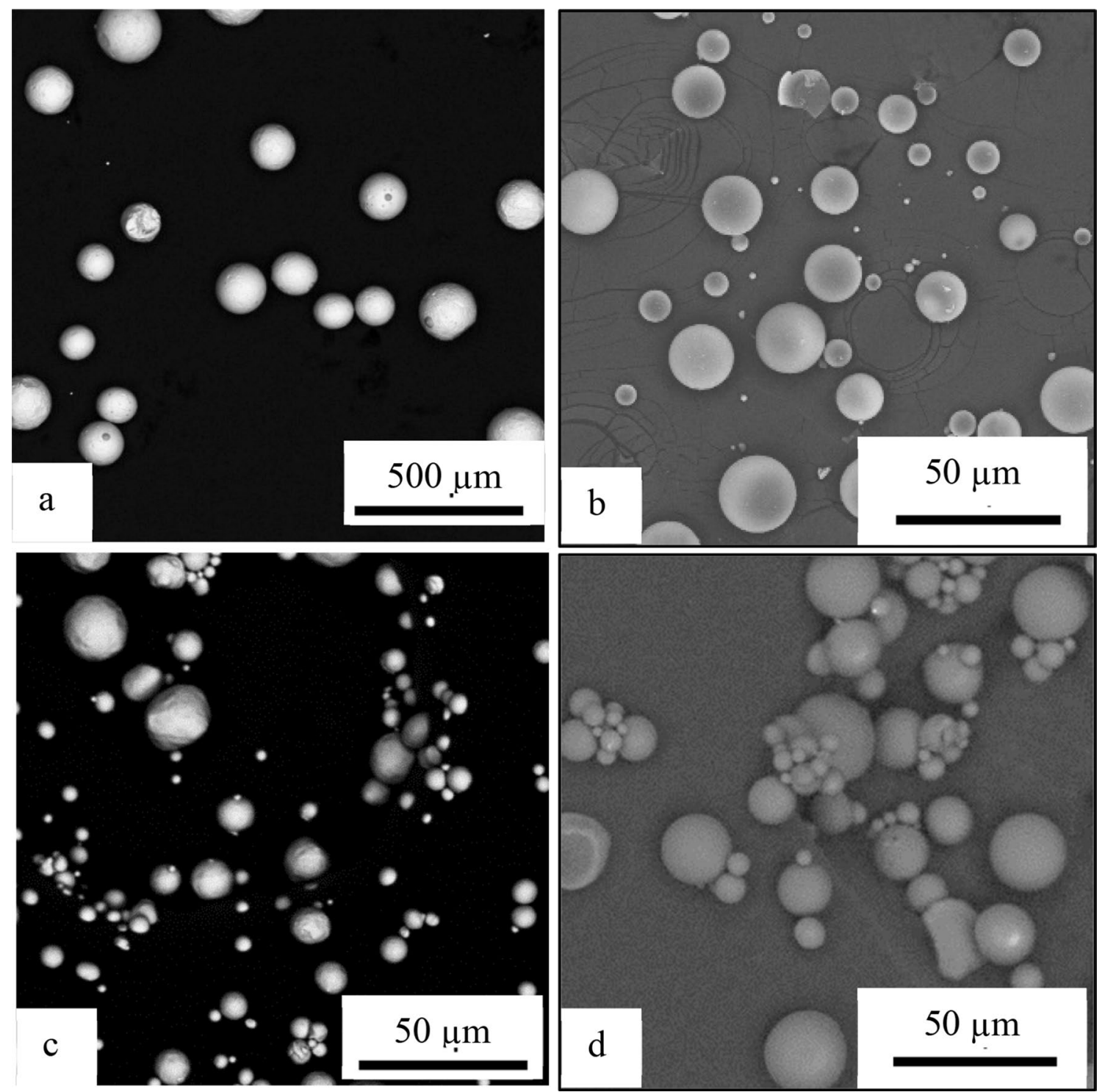

Fig. 2 SEM images of each powder are shown: (a) Bronze, (b) borosilica, (c) copper, (d) porous silica

Table 6 The cubic model b-values calculated for each response and the predicted value based on the optimized composition

\begin{tabular}{lllllllll}
\hline Response & b1 & b2 & b3 & b12 & b13 & b23 & b123 & Predicted values \\
\hline Wet relative density & 0.674 & 1.106 & 1.127 & 0.295 & 0.447 & -0.125 & 3.487 & $118 \%$ \\
Dry relative density & 0.674 & 1.051 & 1.148 & 0.350 & 0.393 & 0.136 & 4.201 & $123 \%$ \\
Sintered relative density & 0.587 & 0.777 & 0.838 & 0.368 & 0.367 & 0.127 & 2.197 & $91.6 \%$ \\
Open porosity & 0.617 & 0.482 & 0.455 & -0.276 & -0.321 & -0.086 & -1.207 & $40.0 \%$ \\
\hline
\end{tabular}

issue. The composition of the binder remained steady up to $2 \mathrm{~h}$ after reaching the working temperature. This indicates that the reservoir was sealed enough to prevent significant amounts of water evaporation during usage. No water additions were therefore necessary during use.

\section{SN Applied Sciences}

\subsection{Injection molding}

\subsubsection{Specimen quality}

For the injection molding of syntactic foams, the binder is a critical part of stopping segregation and promoting 

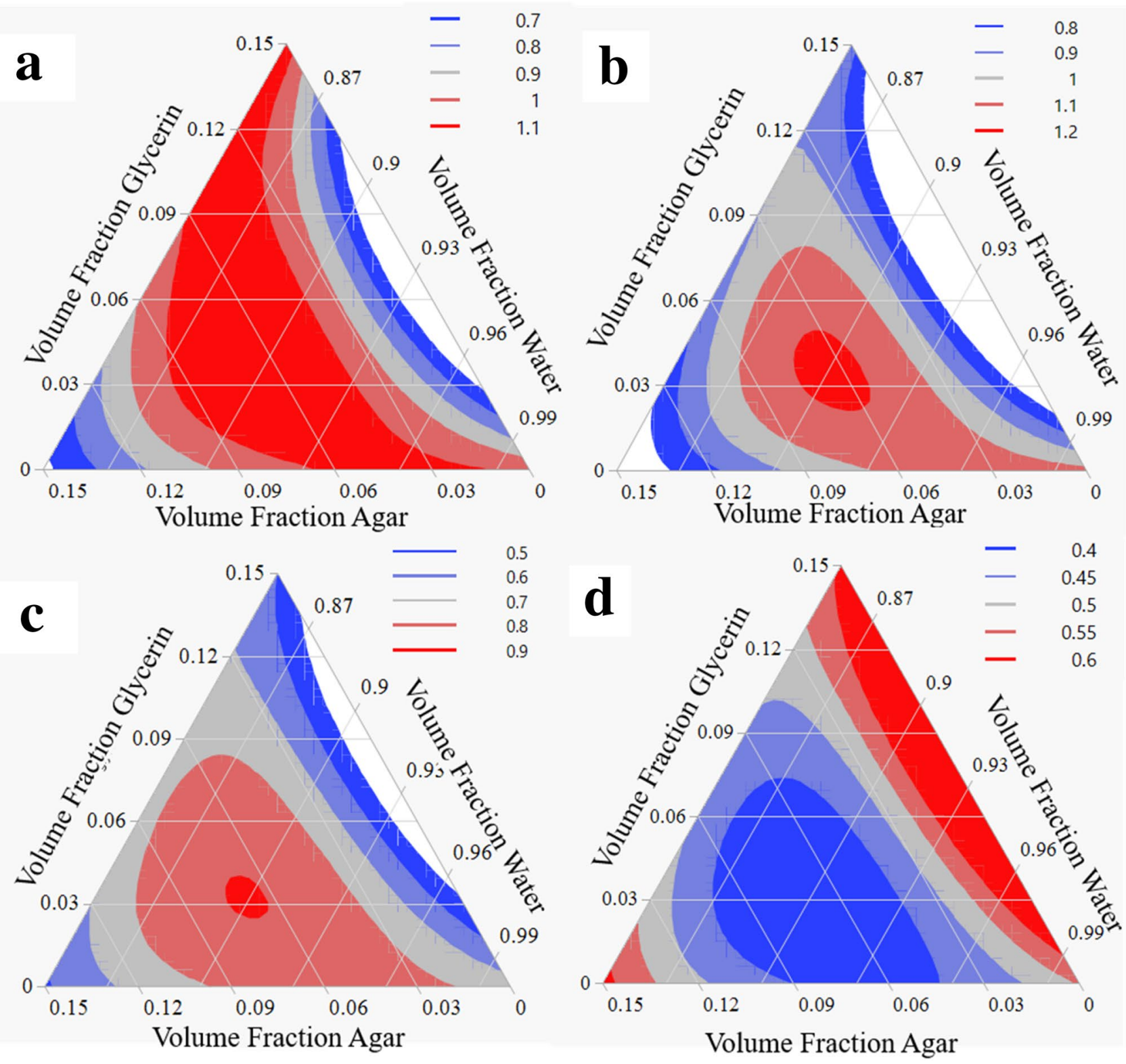

Fig. 3 The response surfaces for the (a) wet density, (b) dry density, (c) bulk sintered density, (d) open porosity after sintering where the legend indicates the relative density fraction for $a, b$ and $c$ and the fraction of open porosity to total volume for $d$

Table 7 The error for each response and relevant variance calculations are tabulated

\begin{tabular}{llllll}
\hline Response & $\mathrm{n}$ & $\mathrm{S}_{\text {pooled }}$ & $\mathrm{S}_{\text {error }}{ }^{2}$ & $\mathrm{~S}_{\mathrm{LF}}{ }^{2}$ & $\mathrm{~F}($ calc) \\
\hline Wet relative density & 3 & 0.029 & 0.010 & 0.002 & 0.159 \\
Dry relative density & 3 & 0.034 & 0.011 & 0.000 & 0.035 \\
Sintered relative density & 3 & 0.029 & 0.010 & 0.002 & 0.159 \\
Open porosity & 3 & 0.0147 & 0.0049 & 0.0003 & 0.0653 \\
\hline
\end{tabular}

good mixing between the dissimilar powders that make up the composite. The way this is determined is by tracking the composition of each specimen after molding. This can then be compared to the input composition of the slurry. The volume and weight of each sample were measured three times: after injection molding (wet), after removing all the water (dry), and after sintering (sintered). These three measurements form the basis of the calculation to determine composition. Appendix shows the calculation steps used to determine the composition for each of the thirty specimens in the samples. 
Fig. 4 The percent mass loss in each binder-only injection molded specimen over time

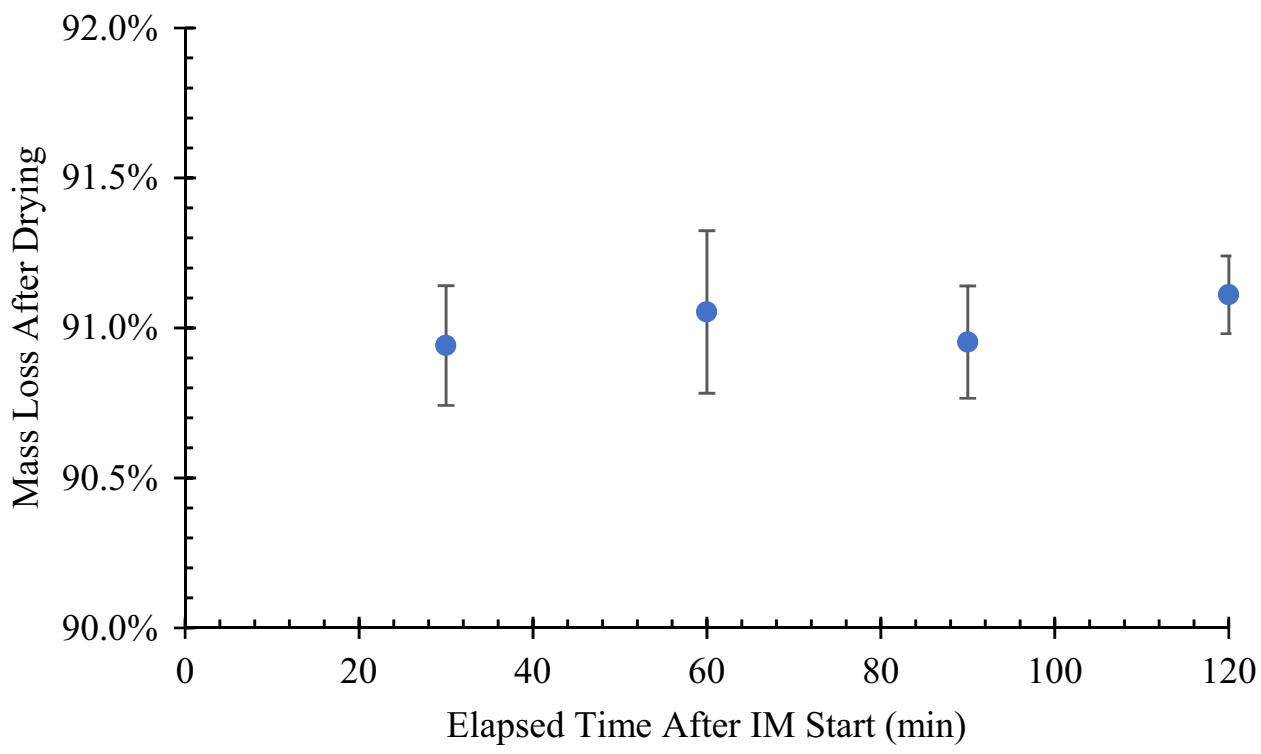

The porosity in the porous silica particles after sintering was not assumed to be the same as before sintering. Instead, this value was found by measuring the average size of the porous silica particles in the composite after sintering and comparing that to the average size of the powder. The AFA data from the feature analysis performed on the $5 \%, 10 \%$, and $15 \%$ P-S specimens were used to determine the average size of the P-S particles after sintering. The average particle size of the porous silica particles after sintering was $9.0 \mu \mathrm{m}$. Compared to the $17.4 \mu \mathrm{m}$ average of the powder before sintering, this change is significantly large. The particles had, on average, $45 \%$ porosity before sintering. The difference in average size after sintering was $52 \%$, so it appears that little to no porosity remained intact in the porous silica spheres. There is significant error in using this technique to estimate the porosity in the silica particles so this may not be accurate. For example, the ASPEX tends to under-predict the size of glass materials. The silica powder was measured manually, in contrast. For this calculation, the sphere porosity was taken as $0 \%$ after sintering.

The results of finding the composition of each specimen are summarized in Table 8. Figures 5 and 6 show the data for each specimen. Figure 5 shows the percentage of binder out of the total wet volume per specimen. Each specimen was assigned a number from 1 to 30 after they were injection molded. The specimen number loosely correlates to time, approximately 1-2 $\mathrm{min} /$ specimen. The binder concentration was relatively stable over time. The amount of binder was generally low compared to what was added to the reservoir (Table 2). The binder may have segregated from the powders in the reservoir or during molding. Figure 6 shows the percentage of
Table 8 The average volume percent of binder and porous silica in each sample after injection molding, as calculated

\begin{tabular}{lll}
\hline Sample & \% binder & \% porous silica \\
\hline $100 \% \mathrm{Cu}$ & $40 \pm 0.6 \%$ & $\mathrm{~N} / \mathrm{A}$ \\
$5 \% \mathrm{P}-\mathrm{S}$ & $40 \pm 2.3 \%$ & $6 \pm 1.9 \%$ \\
$10 \% \mathrm{P}-\mathrm{S}$ & $50 \pm 2.3 \%$ & $11 \pm 3.2 \%$ \\
$15 \%$ P-S & $53 \pm 0.9 \%$ & $24 \pm 3.4 \%$ \\
\hline
\end{tabular}

porous silica in each specimen. The amount of porous silica was high on average, particularly in the case of the $15 \%$ P-S sample. The filler concentration in the $5 \%$ and $10 \%$ P-S samples seemed to fluctuate almost sinusoidally over time. The $15 \%$ P-S specimens did not exhibit this behavior, remaining more consistent except at the ends of the graph. The compositional fluctuations did not appear to be a segregation issue, as in that the composition would have decreased over time.. There may have been pockets of high concentrations of filler that contributed to the fluctuating composition, which would indicate poor mixing of the slurry. Otherwise, it is possible that the slurry segregation was reduced or mitigated by the constant agitation of the slurry in the IM reservoir.

The amount of matrix porosity in the wet specimens was assumed to be negligibly small. However, three specimens from the $5 \%$ P-S sample were found to have significant matrix porosity. These specimens were each found to have a large hole in their center, likely due to an injection molding error. They were removed from the 


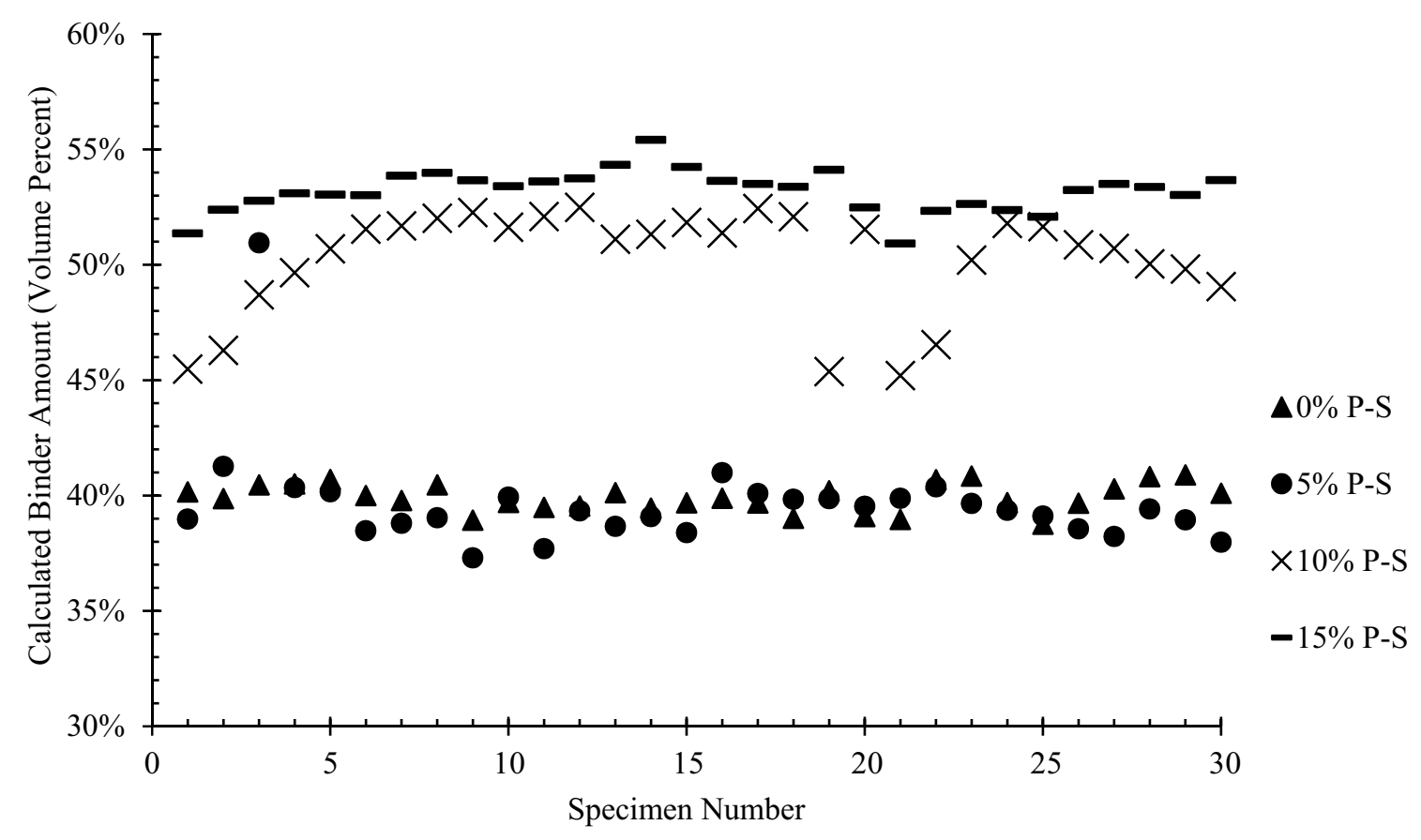

Fig. 5 The percentage of binder in each specimen versus specimen number, where specimen number is the order in which specimens were injection molded

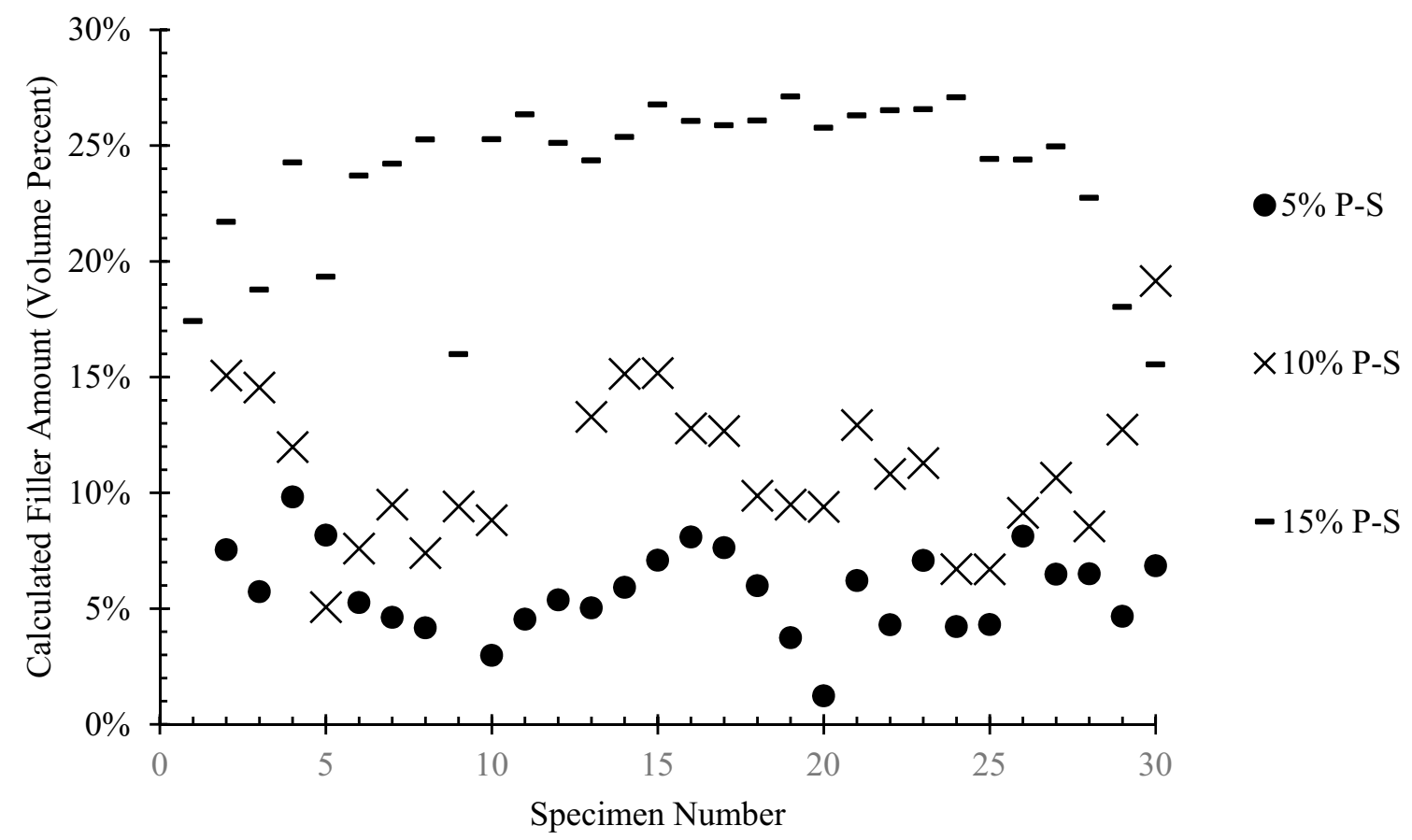

Fig. 6 The percentage of porous silica in each specimen versus specimen number, where specimen number is the order in which specimens were injection molded 
Table 9 The relative density of each sample after each stage of sample post-processing shows the approximate amount of porosity in each sample

\begin{tabular}{lcll}
\hline Sample & $\begin{array}{l}\text { Wet relative density } \\
(\%)\end{array}$ & $\begin{array}{l}\text { Dry relative density } \\
(\%)\end{array}$ & $\begin{array}{l}\text { Sintered } \\
\text { relative } \\
\text { density (\%) }\end{array}$ \\
\hline $100 \%$ Cu & $101 \pm 1.1$ & $64 \pm 0.5$ & $83 \pm 1.3$ \\
$5 \%$ P-S & $99 \pm 3.1$ & $70 \pm 4.9$ & $88 \pm 3.3$ \\
$10 \%$ P-S & $98 \pm 2.3$ & $60 \pm 3.2$ & $82 \pm 2.6$ \\
$15 \%$ P-S & $111 \pm 2.4$ & $70 \pm 2.6$ & $91 \pm 2.3$ \\
\hline
\end{tabular}

data, due to this defect. The other specimens did not appear to have this issue.

\subsection{Binder burnout and sintering}

The relative density of each sample after sintering is shown in Table 9. These are average values calculated from the average filler volume percent of each sample in Table 8. The samples did not sinter to full density despite holding at over $90 \%$ of the melting temperature of copper for $10 \mathrm{~h}$. This is due either to a binder burnout issue or a result of water corrosion affecting the copper powder. Figure 7 shows an element map from a 0\% P-S specimen. This image shows the distribution of copper and oxygen in the specimen. The oxygen seems to surround the porosity, creating a shell of copper oxide. The atmosphere used in this experiment was flowing air. A forming gas would be more appropriate, as it appears the oxygen in the air reacted not just with the carbon in the binder, but also with the copper powder. The formation of copper oxide allowed porosity to remain in the sample during sintering.

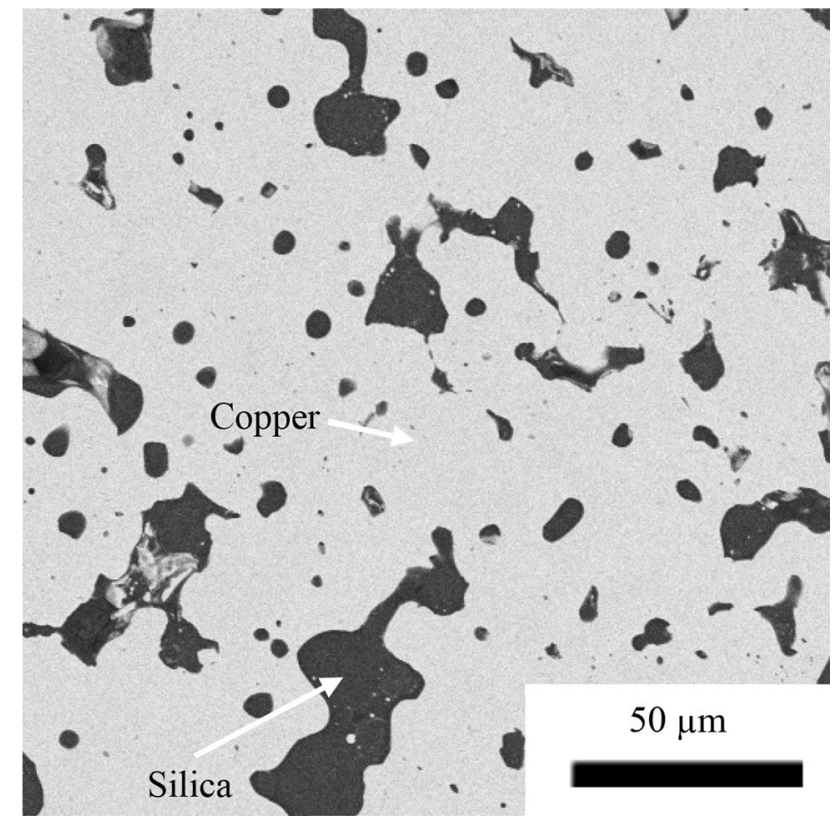

Fig. 8 A micrograph of a 15\% P-S specimen showing the dispersion of the porous silica particles and the morphology of the particles after sintering, the image was taken near the center of a specimen

The amount of carbon present in the samples was found using the ASPEX AFA analysis. One specimen from each sample was tested. The concentration of carbon was $0.11 \% \pm 0.08 \%$ (vol.\%) on average. This indicates that the carbon in the binder was successfully removed from the samples.

The porous silica particles appeared to be well dispersed in the samples. Figure 8 shows a micrograph of a $15 \%$ porous silica specimen. This figure shows the that the particles appear well dispersed. Some of the particles did appear to wick into the surrounding porosity during

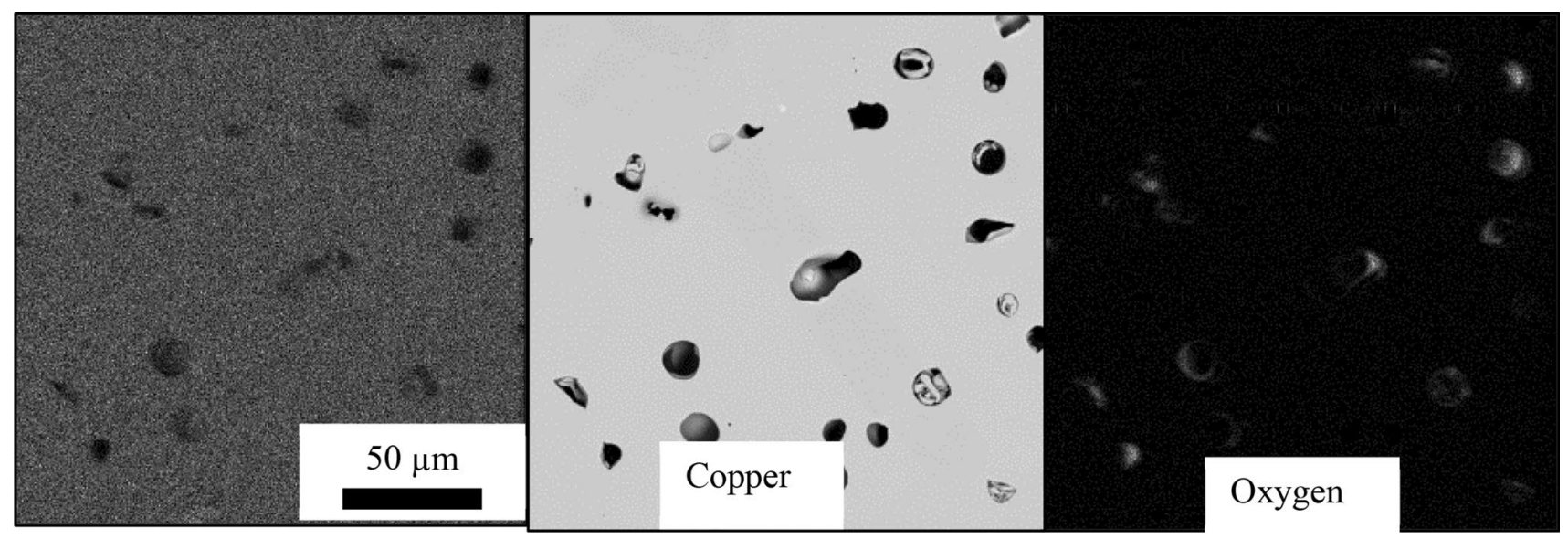

Fig. 7 A element map of a 0\% P-S specimen with the carbon, copper, and oxygen elements identified, the image was taken near the center of a specimen 
sintering. Other particles were surrounded by porosity and remained spherical. Bright spots in the silica may indicate areas of porosity as the edges of the pores in the silica charge under the SEM. Alternatively, these could be copper that migrated into the silica particles. The silica particle did not appear fragmented, most were either spherical or wicked into nearby pores. The well-dispersed particles indicate good local mixing during injection molding.

\subsection{Mechanical testing}

The results of the 3-point bend testing are shown in Fig. 9 compared to other metal matrix syntactic foams. The sources used here use compression yield strength, while the flexural yield strength was measured in this work. The sources also had a variety of material compositions. For these reasons, the yield strengths were not directly compared. Instead, the data was normalized by the matrix properties. In this way, the data is not biased against testing method or material. The graph tracks both the improvement in strength and the decrease in density of each MMSF material over the matrix. The optimal area of the graph for lightweight structural applications is above 1.0 in the strength axis and below 1.0 in the density axis.
This would indicate that the MMSF is stronger and lower in density than the matrix material. The MMSF materials made here met both of these targets, though they are on the low end of both targets.

\section{Conclusions}

Copper and porous silica (P-S) powders were used in a lowpressure injection molding process to make rectangular specimens. Four compositions were made $-0,5,10$, and 15 vol.\% P-S with the remainder being copper. The powders used in this experiment were expected to dry or wet mix poorly due to their similar size and highly dissimilar densities. The binder optimized in this experiment worked well with the syntactic foam materials. The agar-glycerinwater binder was optimized to achieve the highest density. The optimized composition was $7 \%$ agar, $4 \%$ glycerin, and $89 \%$ water. The specimens in each sample composition experienced some compositional fluctuations over time. There was no strong time dependence, however, so this was unlikely to be a segregation issue. The overall average filler concentration was higher than expected for each sample. The binder burnout procedure left $0.11 \% \pm 0.08 \%$

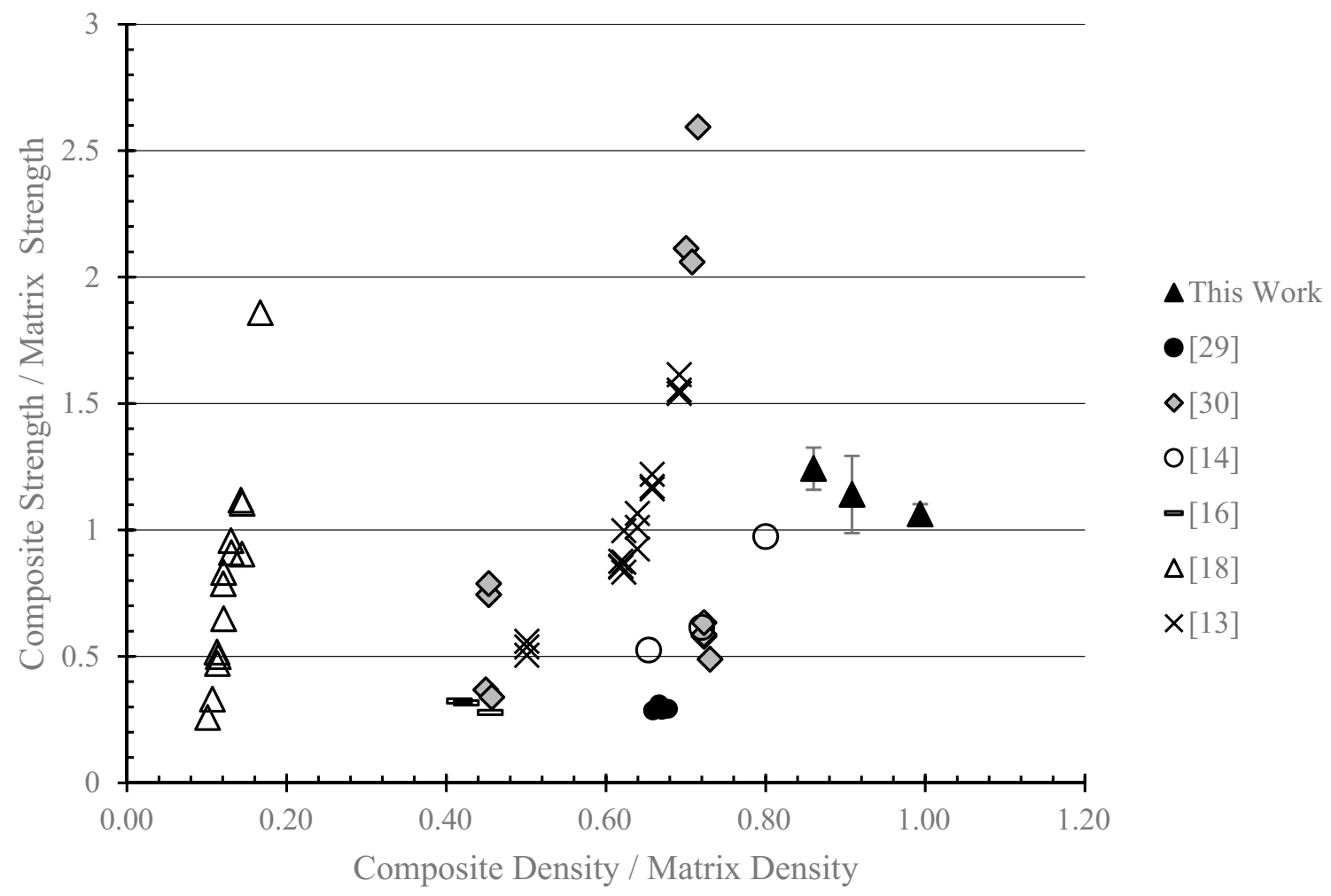

Fig. 9 The strength and density of several MMSF materials from this work and the literature $[13,14,16,18,29,30]$, where each composite's strength was normalized by the average strength of the matrix material and each composite density was normalized by the density of the matrix material for the sake of comparison. Note that some literature values were estimated from figures to the best of the author's ability 
carbon remaining in the specimens, a reasonably low amount. The silica particles were well-dispersed in specimens, indicating good mixing during injection molding. The yield strength of the material was within the optimal range for a lightweight structural composite.

Acknowledgements This research did not receive any specific grant from funding agencies in the public, commercial, or not-for-profit sectors.

\section{Funding None.}

Availability of data and material Can be made available.

\section{Compliance with ethical standards}

Conflict of interest On behalf of all authors, the corresponding author states that there is no conflict of interest.

\section{Appendix}

The concentration of each component in each specimen was required to be calculated. The components were as follows: copper, silica, water, additives, matrix porosity, and sphere porosity. The porous silica contained on average $45 \%$ porosity, and this was kept separate from the matrix porosity. The data used to find these values was the mass and volume measurements at each stage of post-processing. The stages were wet, dry, and sintered. In each stage, the components were different. For example, there was no water in the samples after the drying stage. Equations (A.1)-(A.6) show the equations generated for each mass and volume measurement. The density of each component was also assumed to be known. The density of the components was assumed to be known, per Table 1, and assuming that the porous silica was $45 \%$ porosity, the density of water was $1 \mathrm{~g} / \mathrm{cm}^{3}$ and the density of the additives was also $1 \mathrm{~g} / \mathrm{cm}^{3}$. This calculation also assumes that the pores in the porous silica completely filled with binder during the wet stage. To calculate the volume of sphere porosity after drying, Eqs. (A.7) and (A.8) were used.

$M_{W}=M_{C u}+M_{S M}+M_{\text {water }}+M_{\text {add }}$

$V_{W}=V_{C u}+V_{S M}+V_{\text {water }}+V_{a d d}+V_{W m p}$

$M_{D}=M_{C u}+M_{S M}+M_{a d d}$

$V_{D}=V_{C u}+V_{S M}+V_{a d d}+V_{D m p}+V_{D s p}$

$M_{S}=M_{C u}+M_{S M}$
$V_{S}=V_{C u}+V_{S M}+V_{S m p}$

$\rho_{P S}=\left(\rho_{S M} V_{S M}\right) /\left(V_{T S p}+V_{S M}\right)$

$V_{D s p}=V_{T s p} V_{w} /\left(V_{\text {add }}+V_{w}\right)$

$M, V$, and $\rho$ represent mass, volume, and density, respectively. Subscripts $W, D, S, C u, S M$, water, and add represent wet, dry, sintered, copper, silica material, water, and additives. Xmp and $X s p$ represents matrix porosity and sphere porosity where $X$ can be $W, D, S$, or T (total).

It was assumed that the matrix porosity in subsequent stages depended on the previous stage. Equations (A.9) and (A.10) show these relationships. Notably, all sphere porosity was assumed to be converted to matrix porosity as the sphere porosity collapsed during sintering.

$V_{\text {Dmp }}=V_{\text {water }}+V_{\text {Wmp }}+V_{\text {extra }}$

$V_{S m p}=A *\left(V_{D m p}+V_{T s p} \times V_{A} /\left(V_{\text {add }}+V_{w}\right)+V_{\text {add }}\right)$

$A$ represents the extent to which the matrix densified during the sintering operation. In the $0 \%$ P-S sample, this value was found to be $18.0 \pm 0.807 \%$. This value was assumed to be constant across all specimens. Equations (A.1)-(A.10) were used to calculate the concentration of each component in each injection molded specimen.

\section{References}

1. Gupta N, Rohatgi PK (2014) Metal matrix syntactic foams: processing, microstructure, properties and applications. In: Metal matrix syntactic foams: processing, microstructure, properties and applications. DEStech Publications, Inc., Lancaster, p 370

2. Rohatgi PK, Gupta N, Schultz BF, Luong DD (2011) The synthesis, compressive properties, and applications of metal matrix syntactic foams. JOM 63:36-42

3. Mondal DP, Das S, Jha N (2009) Dry sliding wear behaviour of aluminum syntactic foam. Mater Des 30:2563-2568. https://doi. org/10.1016/J.MATDES.2008.09.034

4. Dou Z, Wu G, Huang $X$ et al (2007) Electromagnetic shielding effectiveness of aluminum alloy-fly ash composites. Compos Part A Appl Sci Manuf 38:186-191. https://doi.org/10.1016/j. compositesa.2006.01.015

5. Szlancsik A, Katona B, Kemény A, Károly D (2019) On the filler materials of metal matrix syntactic foams. Materials (Basel) 12:2023. https://doi.org/10.3390/ma12122023

6. Mondal DP, Das S, Ramakrishnan N, Bhasker KU (2009) Cenosphere filled aluminum syntactic foam made through stir-casting technique. Compos Part A Appl Sci Manuf 40:279-288

7. Agrawal US, Wanjari SP (2017) Physiochemical and engineering characteristics of cenosphere and its application as a lightweight construction material: a review. Mater Today Proc 4:9797-9802. https://doi.org/10.1016/J.MATPR.2017.06.269 
8. Xue X, Zhao Y (2011) Ti matrix syntactic foam fabricated by powder metallurgy: particle breakage and elastic modulus. JOM 63:36-40

9. Lin Y, Zhang Q, Wu G (2016) Interfacial microstructure and compressive properties of $\mathrm{Al}-\mathrm{Mg}$ syntactic foam reinforced with glass cenospheres. J Alloys Compd 655:301-308. https://doi. org/10.1016/j.jallcom.2015.09.175

10. Orbulov I, Májlinger K (2010) On the microstructure of ceramic hollow microspheres. Period Polytech Mech Eng 54:89. https:// doi.org/10.3311/pp.me.2010-2.05

11. Balch DK, Dunand DC (2006) Load partitioning in aluminum syntactic foams containing ceramic microspheres. Acta Mater 54:1501-1511

12. Neville BP, Rabiei A (2008) Composite metal foams processed through powder metallurgy. Mater Des 29:388-396. https://doi. org/10.1016/j.matdes.2007.01.026

13. Májlinger K, Orbulov IN (2014) Characteristic compressive properties of hybrid metal matrix syntactic foams. Mater Sci Eng A 606:248-256. https://doi.org/10.1016/j.msea.2014.03.100

14. Lehmhus D, Weise J, Baumeister J et al (2014) Quasi-static and dynamic mechanical performance of glass microsphere- and cenosphere-based 316 syntactic foams. Procedia Mater Sci 4:383-387. https://doi.org/10.1016/j.mspro.2014.07.578

15. Weise J, Zanetti-Bueckmann V, Yezerska O et al (2007) Processing, properties and coating of micro-porous syntactic foams. Adv Eng Mater 9:52-56. https://doi.org/10.1002/adem.20060 0198

16. Yang Q, Cheng J, Wei Y et al (2020) Innovative compound casting technology and mechanical properties of steel matrix syntactic foams. J Alloys Compd 853:156572. https://doi.org/10.1016/j. jallcom.2020.156572

17. Orbulov IN (2013) Metal matrix syntactic foams produced by pressure infiltration-the effect of infiltration parameters. Mater Sci Eng A 583:11-19. https://doi.org/10.1016/j. msea.2013.06.066

18. Shishkin A, Drozdova M, Kozlov V et al (2017) Vibration-assisted sputter coating of cenospheres: a new approach for realizing Cu-based metal matrix syntactic foams. Metals (Basel) 7:16. https://doi.org/10.3390/met7010016

19. Weise J, Baumeister J, Yezerska O et al (2010) Syntactic iron foams with integrated microglass bubbles produced by means of metal powder injection moulding. Adv Eng Mater 12:604608. https://doi.org/10.1002/adem.200900297
20. Weise J, Lehmhus D, Baumeister J et al (2014) Production and properties of $316 \mathrm{~L}$ stainless steel cellular materials and syntactic foams. Steel Res Int 85:486-497. https://doi.org/10.1002/ srin.201300131

21. German RM, Bose A (1997) Injection molding of metals and ceramics. Metal Powder Industries Federation, Princeton

22. Labropoulos KC, Rangarajan S, Niesz DE, Danforth SC (2004) Dynamic rheology of agar gel based aqueous binders. J Am Ceram Soc 84:1217-1224. https://doi. org/10.1111/j.1151-2916.2001.tb00819.x

23. Sekido $M$, Nakayama $H$, Nukaya $M$, Noro $Y$ (1992) Injection-molding of metal or ceramic powders, US Patent Number US5258155A

24. Zedalis MS, Sherman BC, LaSalle JC (1998) Process for debinding and sintering metal injection molded parts made with an aqueous binder, US Patent Number US5985208A

25. Fanelli AJ, Silvers RD (1986) Process for injection molding ceramic composition employing an agaroid gell-forming material to add green strength to a preform, US Patent Number US4734237A

26. Chen G, Cao P, Wen G et al (2013) Using an agar-based binder to produce porous $\mathrm{NiTi}$ alloys by metal injection moulding. Intermetallics 37:92-99. https://doi.org/10.1016/J.INTER MET.2013.02.006

27. ASTM International (2018) C373-18 standard test methods for determination of water absorption and associated properties by vacuum method for pressed ceramic tiles and glass tiles and boil method for extruded ceramic tiles and non-tile fired ceramic whiteware products

28. ASTM International (2018) C1161-18 standard test method for flexural strength of advanced ceramics at ambient temperature

29. Wang N, Chen X, Li Y et al (2018) Preparation and compressive performance of an A356 matrix syntactic foam. Mater Trans 59:699-705. https://doi.org/10.2320/matertrans.M2018003

30. Rocha Rivero GA, Schultz BF, Ferguson JB et al (2013) Compressive properties of Al-A206/SiC and Mg-AZ91/SiC syntactic foams. J Mater Res 28:2426-2435. https://doi.org/10.1557/ jmr.2013.176

Publisher's Note Springer Nature remains neutral with regard to jurisdictional claims in published maps and institutional affiliations. 